\title{
Breakdown Limits on Gigavolt-per-Meter Electron-Beam-Driven Wakefields in Dielectric Structures
}

\author{
M.C. Thompson,,${ }^{1,2, *}$ H. Badakov, ${ }^{1}$ A. M. Cook, ${ }^{1}$ J. B. Rosenzweig,,${ }^{1}$ R. Tikhoplav, ${ }^{1}$ G. Travish, ${ }^{1}$ I. Blumenfeld, ${ }^{3}$ \\ M. J. Hogan, ${ }^{3}$ R. Ischebeck, ${ }^{3}$ N. Kirby, ${ }^{3}$ R. Siemann, ${ }^{3}$ D. Walz,${ }^{3}$ P. Muggli, ${ }^{4}$ A. Scott,${ }^{5}$ and R. B. Yoder ${ }^{6}$ \\ ${ }^{1}$ Department of Physics and Astronomy, University of California, Los Angeles, California 90095, USA \\ ${ }^{2}$ Lawrence Livermore National Laboratory, Livermore, California 94551, USA \\ ${ }^{3}$ Stanford Linear Accelerator Center, Menlo Park, California 94025, USA \\ ${ }^{4}$ University of Southern California, Los Angeles, California 90089, USA \\ ${ }^{5}$ University of California, Santa Barbara, California 93106, USA \\ ${ }^{6}$ Manhattan College, Riverdale, New York 10471, USA
}

\begin{abstract}
First measurements of the breakdown threshold in a dielectric subjected to $\mathrm{GV} / \mathrm{m}$ wakefields produced by short (30-330 fs), $28.5 \mathrm{GeV}$ electron bunches have been made. Fused silica tubes of $100 \mu \mathrm{m}$ inner diameter were exposed to a range of bunch lengths, allowing surface dielectric fields up to $27 \mathrm{GV} / \mathrm{m}$ to be generated. The onset of breakdown, detected through light emission from the tube ends, is observed to occur when the peak electric field at the dielectric surface reaches $13.8 \pm 0.7 \mathrm{GV} / \mathrm{m}$. The correlation of structure damage to beam-induced breakdown is established using an array of postexposure inspection techniques.
\end{abstract}

Particle accelerators with longitudinal fields orders of magnitude larger than present systems are needed to make continued explorations of particle physics at the energy frontier feasible in the long term [1]. Such advanced accelerators, which promise to dramatically reduce the size and cost of high energy colliders, may also make relativistic beams accessible to smaller laboratories, allowing development of compact light sources such as free-electron lasers and inverse Compton scattering devices [2]. Ultrahigh-field accelerators may not, however, be created from conventional metallic-resonant-cavity-based linear accelerators (linacs) by simply increasing drive power because of breakdown limitations. The relationship between resonant wavelength $\lambda_{r}$ and breakdown field [3] can be used to circumvent this constraint; existing linacs with $\lambda_{r}=$ $1-10 \mathrm{~cm}$ and tens of $\mathrm{MV} / \mathrm{m}$ acceleration gradients should support $\mathrm{GV} / \mathrm{m}$ operation when scaled to $\lambda_{r} \sim 1 \mathrm{~mm}$ and $\mathrm{THz}$ frequencies.

The power levels needed to yield $\mathrm{GV} / \mathrm{m}$ fields in an accelerating structure are available in the IR through optical region of the electromagnetic spectrum from laser sources. Building laser accelerators using resonant structures at the optical scale presents many challenges [4], which are presently being experimentally addressed. These challenges include adherence to unprecedentedly small spatial tolerances in the structure, as well as the injection, propagation, and stable acceleration of beams well below $1 \mu \mathrm{m}$ in both longitudinal and transverse dimensions [5]. It would be advantageous to operate structures in the more forgiving $\mathrm{THz}$ region, but no sources exist at the powers needed to achieve $\mathrm{GV} / \mathrm{m}$ fields.

The need for higher electromagnetic power at short wavelengths has inspired research into beam-driven wake- fields. In such schemes, electromagnetic power is radiated by an ultrashort, intense "driving" electron bunch propagating in a high impedance environment. This power is then used to accelerate another "witness" bunch. With the common enabling component of an ultrashort driving bunch, large amplitude wakefields may be excited in any medium or structure that presents high beam impedance, including resonant dielectric-loaded systems [6] and plasmas [7]. Indeed, with the recent advent of multinanocoulomb, $\sigma_{t}<100$ fs relativistic beams, $50 \mathrm{GV} / \mathrm{m}$ accelerating fields have been produced in an experimental plasma wakefield accelerator [8].

In this Letter, we present a study of the physical limitations encountered driving $>\mathrm{GV} / \mathrm{m}$ wakefields in the simple hollow dielectric tube geometry of a dielectric wakefield accelerator (DWA) [6]; see Fig. 1. In a DWA, an ultrashort drive bunch traverses the evacuated central region of the tube, creating Cherenkov wakefields in the dielectric that propagate outwards at the Cherenkov angle. The Cherenkov fields are then reflected by the dielectric or cladding boundary back towards the center axis where a witness bunch arrives and is accelerated. The DWA approach resolves the $\mathrm{THz}$ source problem by using radiated fields from short electron bunches.

Prior to the present work, wakefields observed in DWAs were limited to long wavelength scales, equivalent to frequencies in the $10 \mathrm{GHz}$ range, and accelerating gradients of at most $100 \mathrm{MV} / \mathrm{m}$, by the length of the multi-ps electron drive beams used $[6,9,10]$. The combination of high charge, short bunch duration, and small spot size available at the Stanford Linear Accelerator Center Final Focus Test Beam (FFTB) facility [11] has enabled the achievement of $\mathrm{GV} / \mathrm{m}$ fields in this experiment; see 


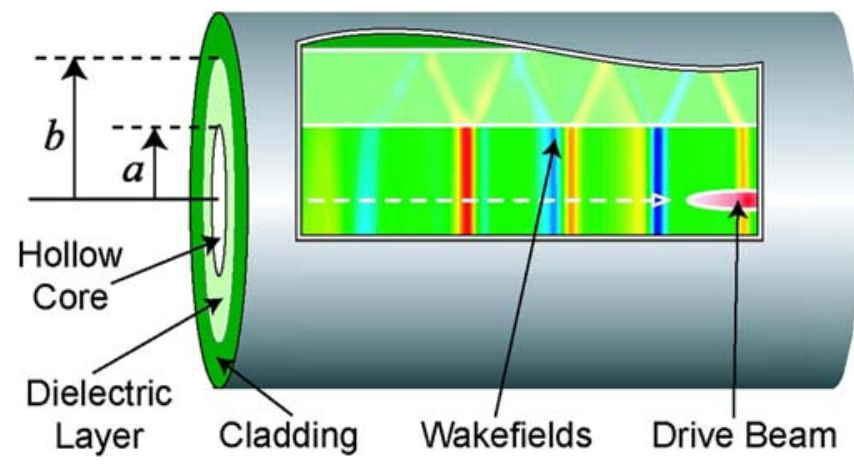

FIG. 1 (color online). Conceptual drawing of the dielectric wakefield accelerator (DWA). A "drive" beam excites wakefields in the tube, while a subsequent witness beam (not shown) would be accelerated by the $E_{z}$ component of the reflected wakefields (bands of color).

Table I. This combination of beam attributes is obtained through both magnetic compression [12], to obtain bunch lengths $<100 \mathrm{fs}$, and the high $28.5 \mathrm{GeV}$ beam energy, which gives small beam sizes naturally through adiabatic damping of the emittance.

The criticality of these beam parameters for driving ultrahigh field wakes in a DWA can be seen from the expression that describes the longitudinal decelerating wakefield within the driving electron beam, $E_{z, \mathrm{dec}}$, and its relation to the peak surface electric field $E_{r \text {,surf }}$. While the formal theory of DWAs is well developed [13-16], a simple treatment based on the classic problem of Cherenkov radiation in the presence of a dielectric boundary [17] yields a more lucid, approximate form for $E_{z, \text { dec }}$ :

$$
e E_{z, \mathrm{dec}}=e E_{r, \mathrm{surf}} \frac{\sqrt{\varepsilon-1}}{\varepsilon} \cong-\frac{4 N_{b} r_{e} m_{e} c^{2}}{a\left[\sqrt{\frac{8 \pi}{\varepsilon-1}} \varepsilon \sigma_{z}+a\right]},
$$

where $e$ is the electron charge, $a$ is the inner radius of the hollow dielectric tube, $\sigma_{z} \cong c \sigma_{t}$ is the rms bunch length, $r_{e}$ and $m_{e} c^{2}$ are the classical radius and rest energy of the electron, respectively, $\varepsilon$ is the dielectric's relative permittivity, and $N_{b}$ is the number of bunch electrons. One could simultaneously obtain the Table I beam parameters at the

TABLE I. Experimental parameters.

\begin{tabular}{lc}
\hline \hline \multicolumn{1}{c}{ Parameter } & Value \\
\hline Dielectric inner diameter $(2 a)$ & $100 \mu \mathrm{m}$ \\
Dielectric outer diameter $(2 b)$ & $324 \mu \mathrm{m}$ \\
Dielectric relative permittivity $(\varepsilon)$ & $\sim 3$ \\
Number of $e^{-}$per bunch $\left(N_{b}\right)$ & $1.4 \times 10^{10}$ \\
RMS bunch length $\left(\sigma_{z}\right)$ & $100-10 \mu \mathrm{m}$ \\
RMS bunch radius $\left(\sigma_{r}\right)$ & $10 \mu \mathrm{m}$ \\
Beam energy & $28.5 \mathrm{GeV}$ \\
Maximum radial field at dielectric surface & $27 \mathrm{GV} / \mathrm{m}$ \\
Maximum decelerating field (vacuum) & $11 \mathrm{GV} / \mathrm{m}$ \\
Maximum accelerating field (vacuum) & $16 \mathrm{GV} / \mathrm{m}$ \\
\hline \hline
\end{tabular}

FFTB. With such small beam dimensions, and $a=$ $50 \mu \mathrm{m}$, decelerating fields up to $11 \mathrm{GV} / \mathrm{m}$ were produced within the beam during these experiments. The peak radial electric field at the dielectric surface is, however, of primary interest in this breakdown study; values as high as $E_{r, \text { surf }}=27 \mathrm{GV} / \mathrm{m}$ were achieved.

While dielectric breakdown has been studied in detail at both optical and $\mathrm{cm}$ wavelengths, only the laser-induced breakdown studies use pulse times comparable to those explored in this experiment. The beam-produced electromagnetic wave contains a fundamental wavelength of $\lambda \cong$ $4(b-a) \sqrt{\varepsilon-1}=634 \mu \mathrm{m}(f=c / \lambda=0.47 \mathrm{THz})$ and higher harmonics. The length of this radiation pulse, as experienced by the downstream end of the tube, is determined by the Cherenkov radiation group velocity and path length through the media. Therefore, while $\sigma_{t}=$ 30-330 fs depending on the level of compression, the pulse of $\mathrm{THz}$ radiation produced by the $1 \mathrm{~cm}$ fused silica tube is always $\sim 100$ ps. Laser-induced breakdown of $\mathrm{SiO}_{2}$ has been studied extensively for wavelengths near $800 \mathrm{~nm}$ and pulse lengths from 20 fs to $7 \mathrm{~ns}[18,19]$. These studies have consistently found a breakdown damage threshold of $\sim 1.1 \mathrm{GV} / \mathrm{m}$ for $100 \mathrm{ps}$ pulses. Thresholds for $\sigma_{t}=$ $30 \mathrm{fs}$ and $330 \mathrm{fs}$ pulses were found to be about $18 \mathrm{GV} / \mathrm{m}$ and $7 \mathrm{GV} / \mathrm{m}$, respectively.

The fundamental mechanism for dielectric breakdown is avalanche ionization. The manner in which avalanche ionization is initiated and driven to the critical density for damage varies with pulse length and photon energy. For long pulse lengths $\gtrsim 10 \mathrm{ps}$, the background carriers dominate the avalanche process and breakdown is insensitive to wavelength [20]. For short pulse lengths $\lesssim 10 \mathrm{ps,}$ multiphoton or tunnel ionization provides the free electrons that lead to the breakdown avalanche [21-23].

Comparisons between this experiment and previous laser breakdown work is complicated by several factors: the relative roles of tunneling and multiphoton ionization, the $\mathrm{THz}$ pulse envelope, and possible background sources of ionizing radiation. While the Keldysh parameter [22] at the fundamental frequency is small, indicating that tunnel ionization is dominant, multiphoton ionization will become increasingly important for the higher harmonics. If the $\mathrm{THz}$ pulse amplitude remains relatively constant for its $\sim 100$ ps duration, breakdown should occur at about $1.1 \mathrm{GV} / \mathrm{m}$ as in the laser experiments at the same pulse length. If, however, the pulse damps rapidly, possibly due to absorption, dispersion, boundary losses, etc., and has a large amplitude for much less than $100 \mathrm{ps}$, then the breakdown field could be higher. It should also be noted that the fused silica in this experiment is subject to additional lowflux sources of ionizing radiation including: incoherent optical and UV Cherenkov photons, stray $28.5 \mathrm{GeV}$ electrons, and background $\mathrm{x}$ rays.

With the above considerations in mind, the experiment carried out at the FFTB was designed to assess the ability of dielectric tubes to withstand the high fields generated by 
short electron bunches. The tubes used in this experiment were produced from commercial, hollow $\mathrm{SiO}_{2}$ fiber optics. Samples of this product were baked to remove the outer polyimide coating, cut into $\sim 1 \mathrm{~cm}$ lengths, polished flat on the ends, and clad with aluminum. Arrays of 10 tubes were placed in precision machined holding blocks. The holders, which were optically prealigned to the nominal electron beam path, could be moved transversely, allowing placement of any of the tubes along the beam axis. A vacuum chamber housed the samples, positioning system, and various beam diagnostics. Tubes of $324 \mu \mathrm{m}$ o.d. and $100 \mu \mathrm{m}$ i.d., along with a few samples with identical o.d. and $200 \mu \mathrm{m}$ i.d., were mounted in the holding blocks. A CCD array camera coupled to a long-working-distance microscope lens was placed in the plane of the holding block to observe the downstream tube end from the side. The digitized microscope-camera images and electron beam parameters were recorded for analysis.

A systematic study of the breakdown threshold of the dielectric tubes was performed. Tubes were monitored for signs of breakdown while the length of the electron bunch passing through them was varied. Except for the energy spread, which controlled the bunch compression, all the other Table I beam parameters, as well as the beam orbit, were held constant. The beam distribution was bi-Gaussian to good approximation. Each fiber was exposed to either a series of fixed length bunches or a ramped set where the bunch length went from minimum to maximum compression over many shots. In all, 20 fibers were each exposed to between 50 and 1000 bunches.

Observations of the visible light output of the fiber ends (see Fig. 2) clearly shows a sudden intensity change when $\sigma_{z}$, and therefore the peak beam current $I_{\text {peak }} \cong$ $c e N_{b} /\left(\sqrt{2 \pi} \sigma_{z}\right)$ crosses a certain threshold value. The light visible in the dim images recorded at long pulse length (and thus low fields) is likely due to incoherent Cherenkov and transition radiation, as well as scintillation. This interpretation is supported by the smooth increase of the lowlevel optical emission as $\sigma_{z}$ was reduced. Once the beam was compressed below about the middle of its bunchlength range, however, the light output jumped up sharply for many of the bunches. These bright emission events were clearly visible on both the side-view microscope camera and a wide-angle top-view observation camera. Furthermore, once below the pulse-length threshold, the occurrence and intensity of a bright flash on a particular shot appeared to be random and uncorrelated to $\sigma_{z}$. No bright-flash events were observed for long bunches with $\sigma_{z}$ above the threshold. Given these characteristics, we identify instances of bright optical emission with plasma discharges produced during dielectric structure breakdown events. Our postexposure inspection of the tubes strongly supports this conclusion.

Unaided visual inspection of the tubes showed dramatic loss of the outer aluminum cladding. The degree of observed aluminum ablation is proportional to the peak beam
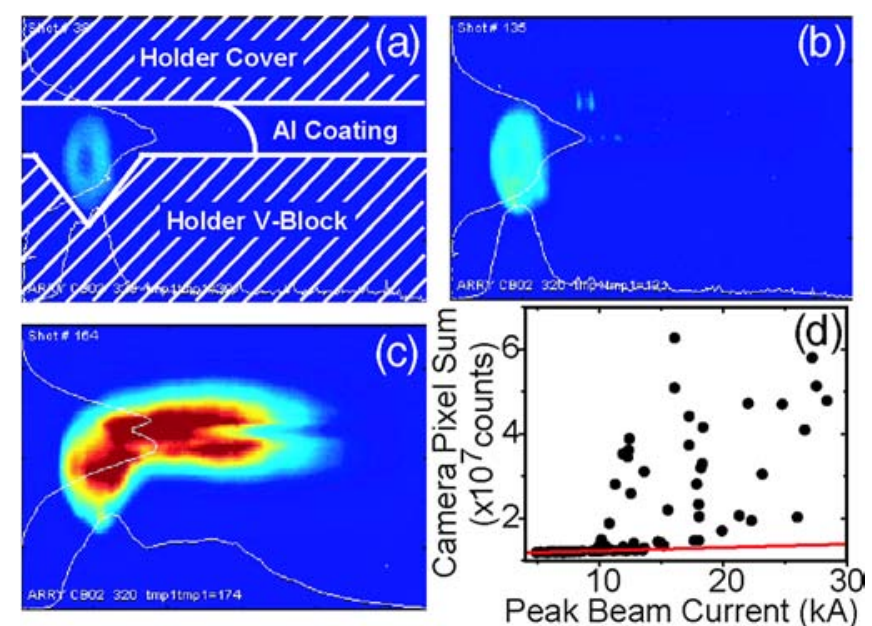

FIG. 2 (color online). Visible (color intensity map) light images of a $100 \mu \mathrm{m}$ i.d. tube for three different $\sigma_{z}$ values (a),(b),(c). In a long pulse case, $\sigma_{z} \sim 45 \mu \mathrm{m}$, the fiber end glows with incoherent optical emission (a). The fiber holder boundaries, viewed at a $45^{\circ}$ angle from beam axis, are also displayed, as is the region where emission is blocked by the fiber's Al coating, which starts $\sim 500 \mu \mathrm{m}$ from the fiber end. For an intermediate pulse length (b) the incoherent optical emissions have increased. In a case of near minimum pulse length (c), $\sigma_{z} \sim$ $12 \mu \mathrm{m}$, a breakdown event produces a large flash of light. This tube's breakdown threshold is visible in a plot (d) of the pixel sum of each frame as a function of $I_{\text {peak }}$. The red line is a fit to the breakdown-free region below $10 \mathrm{kA}$.

current and number of bunches to which a tube was exposed. Calculations indicate that the thin $(\sim 1 \mu \mathrm{m})$, sputtered coatings were vaporized through pulsed Ohmic heating from the beam-induced image currents. As ablation of the metallic cladding was present even in cases without apparent dielectric damage, this process seems to be uncorrelated with breakdown.

Microscope images clearly show structural changes in the tubes that suffered many of the bright-flash events we associate with breakdown; see Fig. 3. These changes include browning of the $\mathrm{SiO}_{2}$, notable roughening of the tube's inner surface, and microcracks in the bulk of the dielectric. The damage level appears to increase with the number of breakdown events a tube experienced. Tubes that were exposed only to non-breakdown-inducing bunches had no visible signs of dielectric damage.

The dielectric tubes also act as optical fibers. Another qualitative evaluation of damage was made by coupling $633 \mathrm{~nm}$ laser light into the dielectric structures through a smaller fiber inserted into the tube bores and observing the transmitted far-field intensity pattern. Samples identified as breakdown damaged through microscopic inspection showed transmission loss, and postfiber far-field intensity-pattern changes, compared to breakdown-free fibers and controls; see Fig. 3. This is expected since the guided-mode intensity of the laser cannot couple effectively into a dielectric with significant surface damage and bulk cracking. Browning also increases absorption. 


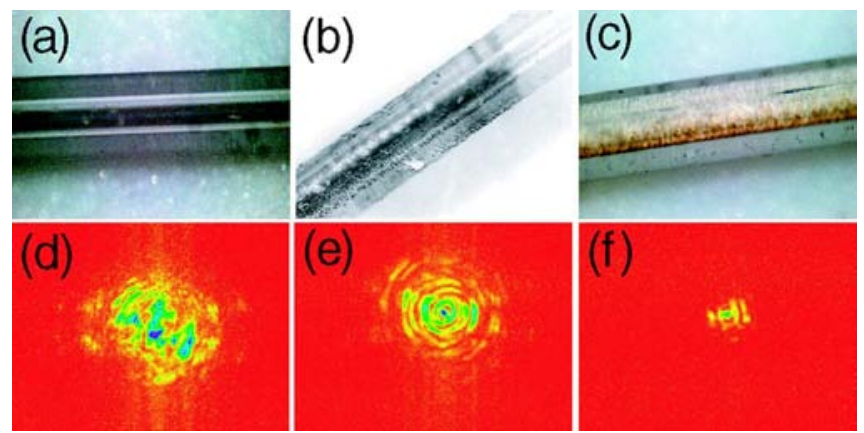

FIG. 3 (color online). Microscope images of select dielectric tubes (a),(b),(c) matched with far-field intensity patterns observed downstream of each laser-illuminated fiber displayed with the same scaled-to-intensity color map (d),(e),(f). The visual appearance and laser transport characteristics of an unexposed control tube (a),(d) compare well to those of a tube exposed to 1000 long bunches (b),(e) for which no breakdown flashes were observed. Alternatively, a tube exposed to 1000 short bunches (c),(f) shows significant changes after suffering numerous breakdown events. These changes include microcracking and radiation-induced browning (c) along with reduced laser transport (f).

All of our observations of the dielectric tubes, both during and after exposure to the electron beam, support the conclusion that the breakdown threshold can be identified by the onset of bright flashes of optical emission during runs where the bunch length was ramped. With the breakdown threshold thus identified, the beam parameter data collected for the corresponding bunch can be analyzed to quantify the breakdown electric field. The first step is to determine $\sigma_{z}$ using a technique previously developed at the FFTB [11]. The energy spectrum of the beam is measured nondestructively by observing synchrotron $\mathrm{x}$ rays produced in a vertical chicane bend magnet. Energy spectra are then uniquely mapped to bunch lengths through comparison with simulations. Once $\sigma_{z}$ has been determined, it is straightforward to combine this knowledge with the measured beam charge and calculate $E_{r, \text { surf }}$ for the breakdown event. This can be done approximately with Eq. (1) or precisely with numerical simulation. The particle-in-cell code OOPIC [24] was used to determine $E_{r \text {,surf }}$ at the dielectric surface on the first breakdown event using the conservative assumption that the beam traveled down the exact center of the structure. The mean of the four most comparable ramped fibers gives a surface field breakdown threshold of $13.8 \pm 0.7 \mathrm{GV} / \mathrm{m}$, which corresponds to a $5.5 \mathrm{GV} / \mathrm{m}$ longitudinal decelerating field within the beam.

In summary, a dielectric surface field breakdown threshold of $13.8 \mathrm{GV} / \mathrm{m}$ has been observed in $1 \mathrm{~cm}$ long, $100 \mu \mathrm{m}$ i.d., $324 \mu \mathrm{m}$ o.d., fused silica DWA structures. This is the highest breakdown field measured in a DWA to date by 2 orders of magnitude. This experiment is also the first time a DWA has been driven at $\mathrm{THz}$ frequencies. It is found that when $\mathrm{SiO}_{2}$ is excited with a fs electron beam, it can sustain fields of roughly the same magnitude as when it is driven by a laser pulse of similar duration. This demonstration of a $13.8 \mathrm{GV} / \mathrm{m}$ breakdown threshold in a specific DWA geometry is a necessary, but not sufficient, condition for the future construction of $\mathrm{GV} / \mathrm{m}$ class DWAs. Future work will examine material fatigue at sub-breakdown fields, wakes in long tubes, direct measurement of accelerating fields, and direct detection of coherent $\mathrm{THz}$ Cherenkov emission.

This work was performed under the auspices of the U.S. Department of Energy under Contracts No. DE-FG0392ER40693, No. DE-AC02-76SF00515, No. W-7405ENG-48, and No. DE-FG02-92-ER40745.

*dr.mcthompson@gmail.com

Present address: Tri Alpha Energy, Inc., Foothill Ranch, CA 92610, USA.

[1] See, for example, C. Joshi and T. Katsouleas, Phys. Today 56, No. 6, 47 (2003).

[2] W. A. Barletta and H. Winick, Nucl. Instrum. Methods Phys. Res., Sect. A 500, 1 (2003).

[3] A. Hassanein et al., Phys. Rev. ST Accel. Beams 9, 062001 (2006).

[4] J. B. Rosenzweig et al., Phys. Rev. Lett. 74, 2467 (1995).

[5] A. Tremaine et al., Phys. Rev. E 56, 7204 (1997).

[6] W. Gai et al., Phys. Rev. Lett. 61, 2756 (1988).

[7] J. B. Rosenzweig et al., Phys. Rev. Lett. 61, 98 (1988).

[8] I. Blumenfeld et al., Nature (London) 445, 741 (2007).

[9] P. Schoessow et al., J. Appl. Phys. 84, 663 (1998).

[10] M.E. Conde, in Proceedings of the 2007 Particle Accelerator Conference, Albuquerque, NM (IEEE, Piscataway, NJ, 2007), p. 1899.

[11] M. J. Hogan et al., Phys. Rev. Lett. 95, 054802 (2005).

[12] P. Emma et al., Stanford Linear Accelerator Center Report No. SLAC-PUB-8850, 2001 (unpublished).

[13] L. Schächter and D. Schieber, Nucl. Instrum. Methods Phys. Res., Sect. A 388, 8 (1997).

[14] M. Rosing and W. Gai, Phys. Rev. D 42, 1829 (1990).

[15] G. Dôme, in Proceedings of the 2nd European Particle Accelerator Conference, Nice, France (Editions Frontieres, Gif-sur-Yvette, France, 1990), p. 628.

[16] K.-Y. Ng, Phys. Rev. D 42, 1819 (1990).

[17] B. M. Bolotovskii, Usp. Fiz. Nauk 75, 295 (1961) [Sov. Phys. Usp. 4, 781 (1962)].

[18] D. Du et al., Appl. Phys. Lett. 64, 3071 (1994).

[19] A.-C. Tien et al., Phys. Rev. Lett. 82, 3883 (1999).

[20] N. Bloembergen, IEEE J. Quantum Electron. QE-10, 375 (1974).

[21] B. C. Stuart et al., J. Opt. Soc. Am. B 13, 459 (1996).

[22] L. V. Keldysh, Sov. Phys. JETP 20, 1307 (1965).

[23] D. M. Simanovskii et al., Phys. Rev. Lett. 91, 107601 (2003).

[24] D. L. Bruhwiler et al., Phys. Rev. ST Accel. Beams 4, 101302 (2001). 